Acta Crystallographica Section D

Biological Crystallography

ISSN 0907-4449

\author{
Alexandre Urzhumtsev, ${ }^{a, b_{*}}$ \\ Pavel V. Afonine ${ }^{\mathrm{C}}$ and \\ Paul D. Adams ${ }^{\mathrm{c}, \mathrm{d}}$
}

a IGBMC, CNRS-INSERM-UdS, 1 Rue Laurent Fries, BP 10142, 67404 Illkirch, France,

${ }^{\mathbf{b}}$ Physics Department, University of Nancy, BP 239, Faculté des Sciences et des

Technologies, 54506 Vandoeuvre-lès-Nancy,

France, ' Lawrence Berkeley National

Laboratory, One Cyclotron Road,

BLDG 64R0121, Berkeley, CA 94720, USA, and

dDepartment of Bioengineering, University of

California Berkeley, Berkeley, CA 94720, USA

Correspondence e-mail: sacha@igbmc.fr

\title{
On the use of logarithmic scales for analysis of diffraction data
}

Predictions of the possible model parameterization and of the values of model characteristics such as $R$ factors are important for macromolecular refinement and validation protocols. One of the key parameters defining these and other values is the resolution of the experimentally measured diffraction data. The higher the resolution, the larger the number of diffraction data $N_{\text {ref }}$, the larger its ratio to the number $N_{\text {at }}$ of non-H atoms, the more parameters per atom can be used for modelling and the more precise and detailed a model can be obtained. The ratio $N_{\text {ref }} / N_{\text {at }}$ was calculated for models deposited in the Protein Data Bank as a function of the resolution at which the structures were reported. The most frequent values for this distribution depend essentially linearly on resolution when the latter is expressed on a uniform logarithmic scale. This defines simple analytic formulae for the typical Matthews coefficient and for the typically allowed number of parameters per atom for crystals diffracting to a given resolution. This simple dependence makes it possible in many cases to estimate the expected resolution of the experimental data for a crystal with a given Matthews coefficient. When expressed using the same logarithmic scale, the most frequent values for $R$ and $R_{\text {free }}$ factors and for their difference are also essentially linear across a large resolution range. The minimal $R$-factor values are practically constant at resolutions better than $3 \AA$, below which they begin to grow sharply. This simple dependence on the resolution allows the prediction of expected $R$-factor values for unknown structures and may be used to guide model refinement and validation.

\section{Introduction}

The maximum resolution of diffraction is an important characteristic of experimental data sets and the resulting crystallographic Fourier synthesis maps. The number of structure factors $N_{\text {ref }}$ for a given crystal depends on the resolution $d$ as

$$
N_{\text {ref }}(d) \simeq d^{-3} \text {. }
$$

Binning of diffraction data, e.g. for the reporting of statistics, can be chosen to be uniform in $\AA$, in $\sin (\theta) / \lambda$, in $\AA^{-1}, \AA^{-2}, \AA^{-3}$ etc. For example, if the resolution limits $d_{k}, k=1,2, \ldots$, are chosen uniformly in $\AA^{-3}$,

$$
\Delta_{-3} d=d_{k}^{-3}-d_{k+1}^{-3}=\text { constant }
$$

moving from $d_{k}$ to $d_{k+1}$ changes the number of reflections approximately by the same amount for all $k$, i.e. equal volumes
Received 17 July 2009

Accepted 29 September 2009
(C) 2009 International Union of Crystallography Printed in Singapore - all rights reserved 
of reciprocal space are covered by each bin. Here, we analyze the effects of partitioning $d_{k}$ uniformly using a logarithmic scale,

$$
\Delta \ln d=\ln \left(d_{k+1}\right)-\ln \left(d_{k}\right)=\text { constant. }
$$

In this case, moving from $d_{k}$ to $d_{k+1}$ changes the number of reflections by approximately the same factor. Using this regime, we can perform analyses to establish whether selected crystallographic characteristics have a simple dependence on resolution on this logarithmic scale. One such characteristic is the ratio of the number of diffraction data $N_{\text {ref }}$ to the number $N_{\text {at }}$ of atoms for structures solved at a given resolution. Ideally, the total number of parameters of a model should not exceed the number of independent observations (reflections) or the model is considered to be overparametrized and inappropriate for refinement. Therefore, the typical value of $N_{\text {ref }} / N_{\text {at }}$ at a given resolution indicates the allowed number of parameters per atom and therefore defines a 'typical model' at this resolution. Knowledge of this ratio can also help to predict the number of molecules per unit cell. Inversely, for a known Matthews coefficient (Matthews, 1968),

$$
V_{\mathrm{M}}=V M_{\mathrm{w}}^{-1} N_{\mathrm{sym}}^{-1},
$$

it may help to estimate the expected high-resolution diffraction limit of the crystal as discussed below, thus completing other indicators (see, for example, Arai et al., 2004, and references therein), in particular the overall $B$ value (Wilson, 1949). Here, $V$ is the unit-cell volume, $N_{\text {sym }}$ is the number of crystallographic symmetry operations and $M_{\mathrm{w}}$ is the molecular weight of the macromolecules in the asymmetric part of the unit cell.

Expected 'typical' values of the crystallographic $R$ factor, of the $R_{\text {free }}$ value (Brünger, 1992) and of their difference are often considered during structure solution. To our knowledge, despite numerous studies (for example, Luzzati, 1952; Cruickshank, 1996; Brünger, 1997; Tickle et al., 1998, 2000; Read \& Kleywegt, 2009; Urzhumtseva et al., 2009; Joosten et al., 2009), a convenient and simple analytic expression for the $R$ factors typical at a given resolution is still not well defined. We used a logarithmic scale to study these functions and also the minimal values of the $R$ factor. The latter can be considered as a goal that in most cases can be achieved at a given resolution.

Summarizing, the goal of this study was to determine whether an appropriate choice of resolution binning using different scales highlights a simple analytic dependence of macromolecular model characteristics. Knowledge of such a dependence can help in structure solution and can be used as an auxiliary validation criterion.

\section{Test data and parameters}

We selected models from the PDB (Bernstein et al., 1977; Berman et al., 2000; selection in March 2009) for which the database contained experimental data: 31662 entries in total (set 1). For these models we extracted the characteristics as they were reported in the file headers. Two subsets (sets 2 and 3), with 29484 and 710 entries, respectively, consisted of models of proteins only and models that included nucleic acids.

Independently, a number of crystallographic characteristics, including $R$ factors, were recalculated using the phenix.model vs_data (Afonine et al., in preparation) utility of PHENIX (Adams et al., 2002). Set 4 consisted of 30546 entries, which were those of set 1 excluding obvious outliers as indicated by $R$ factor. Set 5 consisted of entries for which a test set was available allowing the calculation of $R_{\text {free }}$ factors and contained 22504 entries in total. Details of these data sets are given below.

For our uniform logarithmic grid we needed to define its step and origin. We chose the step $\Delta \ln d$ such that from one resolution limit to another the number of reflections changed by a factor of 1.5. [It follows from equations 1 and 3 that $\Delta \ln d=\frac{1}{3} \ln (1.5) \simeq 0.135$.] Also, for convenience of presentation we chose the origin $d_{1}=\frac{2}{3} \AA$ such that the resolution $d=1.0 \AA(\ln d=0.0)$ falls exactly at a grid node.

\section{Number of data per atom}

\subsection{Preliminary analysis for selected data sets}

As mentioned above, the ratio $N_{\text {ref }} / N_{\text {at }}$, the ratio of the number of independent reflections $N_{\text {ref }}$ to the number $N_{\text {at }}$ of independent macromolecular non- $\mathrm{H}$ atoms in the unit cell, is important in helping to define the possible parameterizations of an atomic model when working with diffraction data at a given resolution. The total number of reflections at a given resolution $d$ can be expressed through the volumes $V$ and $V^{*}$ of the unit cell in direct or reciprocal space, respectively, as

$$
N_{\text {ref }}^{\text {full }} \simeq \frac{4 \pi}{3} d^{-3}\left(V^{*}\right)^{-1} \simeq \frac{4 \pi}{3} d^{-3} V .
$$

When the structure factors obey Friedel's law, for a given crystal the dependence on resolution is

$$
N_{\text {ref }} / N_{\mathrm{at}} \simeq\left(\frac{1}{2} N_{\mathrm{ref}}^{\mathrm{full}} N_{\mathrm{sym}}^{-1}\right) N_{\mathrm{at}}^{-1} \simeq \frac{2 \pi}{3} d^{-3} V_{\mathrm{M}} M_{\mathrm{w}} N_{\mathrm{at}}^{-1} \simeq \eta d^{-3} V_{\mathrm{M}}
$$

(otherwise the coefficient $\frac{1}{2}$ would be absent). For protein structures, the mean ratio $M_{\mathrm{w}} N_{\mathrm{at}}^{-1}$ can be approximately estimated from the molecular weight and atom content of different residues, resulting in the coefficient $\eta=$ $(2 \pi / 3) M_{\mathrm{w}} N_{\mathrm{at}}^{-1} \simeq 27$.

We calculated the ratio $N_{\text {ref }} / N_{\text {at }}$ for all models of set 1 . Here, $N_{\text {at }}$ is the number of non- $\mathrm{H}$ atoms in the PDB model and $N_{\text {ref }}$ is equal to the number of reflections in the deposited file; anomalous pairs of reflections, which are highly correlated, were considered as a single reflection when presented (in 1051 data sets). In our study, we characterize the structure by the resolution $d_{\mathrm{PDB}}$ at which the deposited structure has been reported. Obviously, this characteristic depends on a number of subjective factors such as the accepted completeness of the highest resolution zone, particular experimental conditions and restrictions etc. However, the large number of structures available from the current PDB for our analysis minimizes any 
systematic bias arising from these factors. Our first goal was to determine whether the dependence of the calculated $N_{\text {ref }} / N_{\text {at }}$ and reported $d_{\mathrm{PDB}}$ reflects relation (6). Fig. 1(a) shows the distribution of $\ln N_{\text {ref }} / N_{\text {at }}$ versus resolution $d_{\mathrm{PDB}}$ on a uniform logarithmic scale for a subset of models with data completeness above $99 \%$ and a Matthews coefficient of $2.35<V_{\mathrm{M}}<$ $2.45 \AA^{3} \mathrm{Da}^{-1}$, close to the typical value for $V_{\mathrm{M}}$ of $2.4 \AA^{3} \mathrm{Da}^{-1}$. $V_{\mathrm{M}}$ was taken from the PDB headers; the selection gave 313 models. The points fitted well to a straight line. Two obvious outliers correspond to the models $2 \mathrm{v} 5 \mathrm{k}$ and $1 \mathrm{yqn}$, for which the deposited atoms correspond to one half and one third of the whole cell content, respectively, owing to corresponding local (noncrystallographic) symmetries. When these symmetries were taken into account, the points fitted closely to the line (see, for example, the case of 1yqn indicated by an arrow in Fig. 1a).

The slope of the straight line differs slightly from -3 as expected from (6). We supposed that some differences might be found in the reported $V_{\mathrm{M}}$ values. For example, at high resolution some authors may include $\mathrm{H}$ atoms, differently from at lower resolutions; conversely, at low resolutions one might miss the contribution of disordered parts or side chains that are invisible in maps and absent from the model. To study this issue, we recalculated the $V_{\mathrm{M}}$ value for all reported structures considering the full macromolecular content of the cell according to the deposited sequence. Obviously, this recalculation modified the set of selected models (291 models with $2.35<V_{\text {M-calc }}<2.45 \AA^{3} \mathrm{Da}^{-1}$ ).
When the PDB-reported $V_{\mathrm{M}}$ values were substituted by the recalculated values, the plot of $\ln N_{\text {ref }} / N_{\text {at }}$ had the expected slope (Fig. 1b). This observation also gave us confidence that there was no significant discrepancy between the resolution limits $d_{\mathrm{PDB}}$ in the PDB-reported structures and that further analysis could be based on these values.

A similar $\ln N_{\text {ref }} / N_{\text {at }}$ versus $d_{\text {PDB }}$ distribution for all models with $2.35<V_{\text {M-calc }}<2.45 \AA^{3} \mathrm{Da}^{-1}$ (Fig. 1 ; $; 2754$ models) contains several points that are below this line owing to incomplete data sets. The data completeness 'compl' was then taken into account so that in further calculations $N_{\text {ref }}$ corresponded to a complete set of data as measured at a reported resolution $d_{\mathrm{PDB}}, N_{\mathrm{ref}}^{\text {full }}=N_{\mathrm{ref}} \mathrm{compl}^{-1}$. This new distribution (Fig. 1d) has the same features as that in Fig. 1(b) but is more significant statistically. In general, correcting for completeness instead of rejecting models with incomplete data sets makes the set of models much more representative. In particular, crystals with strongly anisotropic diffraction patterns can be studied together with isotropically diffracting structures with no need for the introduction of artificial selections.

When we analyze the distribution of $\ln N_{\text {ref }} / N_{\text {at }}$ for all PDB entries with compl $>99 \%$ we observe that the corresponding cloud of points is larger but still essentially linear (Fig. 1e; 4020 models). However, the slope of the principal axis is now significantly lower than previously calculated. Kantardjieff \& Rupp (2003) studied the dependence of $V_{\mathrm{M}}$ on different factors and in particular showed that the mean $V_{\mathrm{M}}$ increases with resolution; according to (6) this explains the lower slope

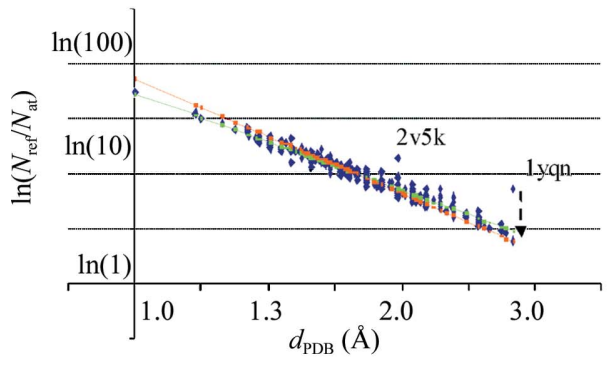

(a)

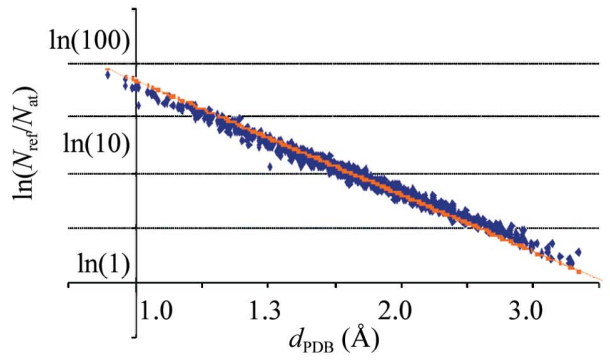

(d)

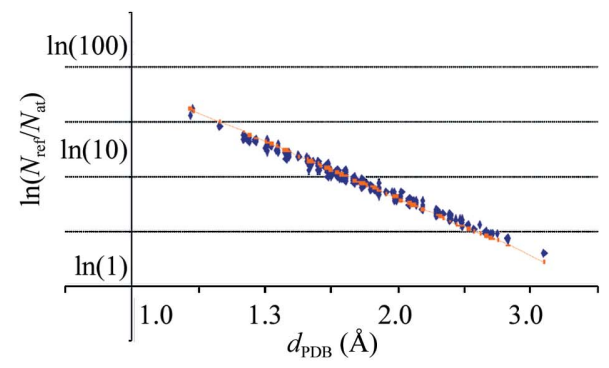

(b)

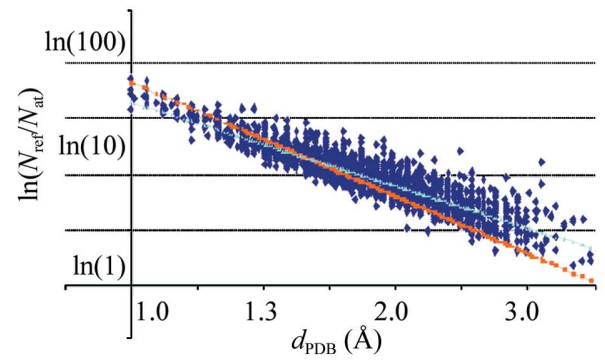

(e)

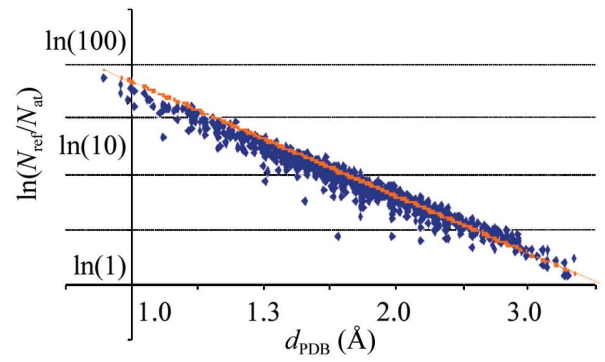

(c)

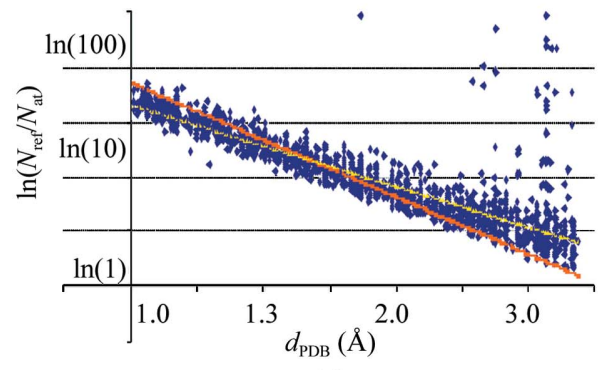

$(f)$

Figure 1

Distribution of the $\ln \left(N_{\mathrm{ref}} / N_{\mathrm{at}}\right)$ value versus resolution $d_{\mathrm{PDB}}$ on a uniform logarithmic scale. (a) Structures with Matthews coefficient $2.35<V_{\mathrm{M}}<$ $2.45 \AA^{3} \mathrm{Da}^{-1} . V_{\mathrm{M}}$ is taken from the file headers and data completeness is above $99 \%$. The broken arrow shows the change in the ratio after a correct assignment of $N_{\text {at }}$ for 1yqn. (b) The same as $(a)$ but with $V_{\mathrm{M}}$ recalculated. (c) The same as $(b)$ but without selection of entries by data completeness. $(d)$ The same as $(c)$ but with correction for data completeness. $(e)$ All models with data completeness above $99 \%$. $(f)$ Random selection from the whole PDB with correction for data completeness. The orange line corresponds to theoretical values for crystals with $V_{\mathrm{M}}=2.4 \AA^{3} \mathrm{Da}^{-1}$. The green, blue and yellow lines show the linear approximations for $(a),(e)$ and $(f)$, respectively. See text for details. 
Table 1

Number of models in different sets used for statistics.

Columns 3 and 4 show the median of the intervals in angstroms and on a logarithmic scale. See text for descriptions of the data sets.

\begin{tabular}{|c|c|c|c|c|c|c|c|c|}
\hline$\underline{N}$ & $\begin{array}{l}\text { Resolution } \\
\text { shell }\left(d_{1}-d_{2}\right) \\
(\AA) \\
\end{array}$ & $\begin{array}{l}\text { Median } \\
\left(d_{1} d_{2}\right)^{1 / 2} \\
(\AA)\end{array}$ & $\begin{array}{l}\text { ln-median } \\
\frac{1}{2} \ln \left(d_{1} d_{2}\right)\end{array}$ & $\begin{array}{l}\text { Set } 1 \\
\text { (with } F_{\text {obs }} \text { ) }\end{array}$ & $\begin{array}{l}\text { Set } 2 \\
\text { (no nucleic } \\
\text { acids) }\end{array}$ & $\begin{array}{l}\text { Set } 3 \\
\text { (nucleic } \\
\text { acids) }\end{array}$ & $\begin{array}{l}\text { Set } 4 \\
\left(17.0>R_{\mathrm{PDB}}\right. \\
>0.06)\end{array}$ & $\begin{array}{l}\text { Set } 5 \\
\text { (with test } \\
\text { data set) } \\
\end{array}$ \\
\hline 1 & $<0.67$ & & & 3 & 3 & 0 & 3 & 0 \\
\hline 2 & $0.67-0.76$ & 0.71 & -0.338 & 8 & 7 & 1 & 7 & 3 \\
\hline 3 & $0.76-0.87$ & 0.82 & -0.206 & 42 & 41 & 1 & 38 & 12 \\
\hline 4 & $0.87-1.00$ & 0.93 & -0.070 & 196 & 178 & 16 & 177 & 76 \\
\hline 5 & $1.00-1.14$ & 1.07 & 0.066 & 336 & 312 & 22 & 319 & 167 \\
\hline 6 & $1.14-1.31$ & 1.22 & 0.201 & 729 & 687 & 34 & 687 & 432 \\
\hline 7 & $1.31-1.50$ & 1.40 & 0.338 & 1878 & 1794 & 67 & 1807 & 1230 \\
\hline 8 & $1.50-1.72$ & 1.61 & 0.474 & 3639 & 3459 & 119 & 3470 & 2592 \\
\hline 9 & $1.72-1.97$ & 1.84 & 0.610 & 6574 & 6329 & 90 & 6248 & 4676 \\
\hline 10 & $1.97-2.25$ & 2.11 & 0.744 & 7169 & 6790 & 132 & 6916 & 5211 \\
\hline 11 & $2.25-2.58$ & 2.41 & 0.879 & 5385 & 4992 & 103 & 5256 & 4001 \\
\hline 12 & $2.58-2.95$ & 2.76 & 1.015 & 3821 & 3355 & 84 & 3767 & 2823 \\
\hline 13 & $2.95-3.37$ & 3.15 & 1.148 & 1451 & 1222 & 37 & 1428 & 1039 \\
\hline 14 & $3.37-3.86$ & 3.61 & 1.283 & 310 & 232 & 4 & 308 & 190 \\
\hline 15 & $3.86-4.42$ & 4.13 & 1.418 & 82 & 64 & 0 & 78 & 44 \\
\hline 16 & $4.42-5.06$ & 4.73 & 1.554 & 16 & 9 & 0 & 15 & 3 \\
\hline 17 & $5.06-5.80$ & 5.42 & 1.690 & 6 & 3 & 0 & 5 & 1 \\
\hline 18 & $5.80-6.63$ & 6.20 & 1.825 & 5 & 1 & 0 & 5 & 1 \\
\hline 19 & $6.63-7.59$ & 7.09 & 1.959 & 6 & 5 & 0 & 6 & 1 \\
\hline 20 & $7.59-8.69$ & 8.12 & 2.095 & 1 & 1 & 0 & 1 & 1 \\
\hline \multirow[t]{2}{*}{21} & $8.69-9.95$ & 9.30 & 2.230 & 5 & 2 & 0 & 5 & 1 \\
\hline & Total & & & 31662 & 29486 & 710 & 30546 & 22504 \\
\hline
\end{tabular}

Table 2

Statistical information for $N_{\text {ref }} / N_{\text {at }}$ in the resolution shells chosen uniformly on a logarithmic scale.

Columns 2 and 3 give the PDB codes for the protein structures with the minimal and maximal value of the ratio. Columns 7 and 8 show the values of the linear interpolations in the resolution interval $(0.76,2.58 \AA)$ (see Table 3). The last column gives the difference of the modes calculated for sets 1 and 2 of the models.

\begin{tabular}{|c|c|c|c|c|c|c|c|c|}
\hline \multirow[b]{2}{*}{$\begin{array}{l}\text { Resolution } \\
\text { shell }(\AA)\end{array}$} & \multicolumn{2}{|c|}{ PDB code } & \multicolumn{3}{|c|}{$N_{\text {ref }} / N_{\text {at }}$} & \multicolumn{3}{|c|}{ Linear interpolation } \\
\hline & $\begin{array}{l}\text { Min. } \\
N_{\text {ref }} / N_{\text {at }}\end{array}$ & $\begin{array}{l}\text { Max. } \\
N_{\text {ref }} / N_{\text {at }}\end{array}$ & Min. & Max. & Mean & Mean & Mode & $\begin{array}{l}\text { Mode difference } \\
\text { set } 1 / \text { set } 2\end{array}$ \\
\hline$<0.67$ & $2 \mathrm{vb} 1$ & 1ucs & 124.8 & 178.4 & 152.9 & 149.0 & 130.7 & \\
\hline $0.67-0.76$ & $1 \mathrm{r} 6 \mathrm{j}$ & $1 \mathrm{yk} 4$ & 88.1 & 253.0 & 133.5 & 109.1 & 96.8 & \\
\hline $0.76-0.87$ & $1 \mathrm{~m} 40$ & $1 \mathrm{n} 55$ & 50.6 & 180.7 & 81.7 & 79.8 & 71.7 & -0.01 \\
\hline $0.87-1.00$ & $2 \mathrm{gkg}$ & $2 \mathrm{rbk}$ & 39.0 & 106.1 & 57.0 & 58.4 & 53.1 & 0.06 \\
\hline $1.00-1.14$ & $2 \mathrm{ofm}$ & $1 \mathrm{rqw}$ & 26.2 & 113.1 & 45.2 & 42.8 & 39.3 & -0.16 \\
\hline $1.14-1.31$ & 2qj7 & $2 \mathrm{dlb}$ & 18.9 & 90.7 & 31.9 & 31.3 & 29.1 & 0.00 \\
\hline $1.31-1.50$ & $106 \mathrm{v}$ & 2ew0 & 12.8 & 54.3 & 21.8 & 22.9 & 21.5 & 0.00 \\
\hline $1.50-1.72$ & $20 \mathrm{mq}$ & 2 dga & 8.1 & 56.6 & 15.8 & 16.8 & 15.9 & 0.00 \\
\hline $1.72-1.97$ & 3 ins & $2 \operatorname{egx}$ & 5.6 & 40.5 & 11.8 & 12.3 & 11.8 & -0.02 \\
\hline $1.97-2.25$ & $1 \mathrm{e} 0 \mathrm{p}$ & $1 \mathrm{zba}$ & 3.8 & 292.5 & 9.1 & 9.0 & 8.7 & 0.00 \\
\hline $2.25-2.58$ & 2ins & $2 \mathrm{izw}$ & 2.8 & 565.7 & 7.0 & 6.6 & 6.5 & 0.00 \\
\hline $2.58-2.95$ & $2 p 3 c$ & 1ng0 & 2.4 & 465.2 & 6.1 & 4.8 & 4.8 & -0.02 \\
\hline $2.95-3.37$ & $2 \mathrm{vdt}$ & $1 d w n$ & 1.8 & 694.6 & 7.4 & 3.5 & 3.5 & 0.00 \\
\hline $3.37-3.86$ & $2 \mathrm{dc} 3$ & $1 \mathrm{c} 8 \mathrm{~h}$ & 1.5 & 293.1 & 8.7 & 2.6 & 2.6 & 0.00 \\
\hline $3.86-4.42$ & $2 \mathrm{gsz}$ & $1 \times 35$ & 1.1 & 73.0 & 3.9 & 1.9 & 1.9 & 0.01 \\
\hline $4.42-5.06$ & 1 ye1 & $2 \mathrm{~g} 34$ & 0.9 & 89.5 & 12.7 & 1.4 & 1.4 & \\
\hline $5.06-5.80$ & $3 b 5 x$ & $2 \mathrm{gp} 1$ & 8.1 & 32.2 & 16.2 & 1.0 & 1.1 & \\
\hline $5.80-6.63$ & $2 z q p$ & $2 z q p$ & 0.8 & 0.8 & 0.8 & 0.7 & 0.8 & \\
\hline $6.63-7.59$ & $3 \mathrm{c} 4 \mathrm{y}$ & $1 \mathrm{yv} 0$ & 0.3 & 0.6 & 0.5 & 0.5 & 0.6 & \\
\hline $7.59-8.69$ & $2 \mathrm{dh} 1$ & $2 \mathrm{dh} 1$ & 2.6 & 2.6 & 2.6 & 0.4 & 0.4 & \\
\hline $8.69-9.95$ & $1 \mathrm{vcr}$ & $2 \mathrm{qzv}$ & 3.2 & 14.1 & 8.6 & 0.3 & 0.3 & \\
\hline
\end{tabular}

we observed. An alternative calculation without selection by compl $>99 \%$ but using the completeness-corrected number of reflections $N_{\text {ref }}^{\text {full }}$ as above showed a similar distribution (Fig. $1 f$; for illustration purposes we selected randomly 250 models per resolution shell; shells with less than 200 models were excluded; 2489 models in total).

\subsection{Maximum-mean-minimum analysis}

To analyze the features of the distributions obtained in \$3.1, we studied them in more detail as described below. Our goal was to find a simple dependence of the principal statistical characteristics of $N_{\text {ref }} / N_{\text {at }}$ as a function of resolution. Following Kantardjieff \& Rupp (2003), in order to work with a more homogenous set of models we excluded all entries containing nucleic acids. This left us with 29486 entries (set 2; Table 1). In order to have a sample size that was as large as possible we did not reject incomplete data sets but, in accordance with preliminary analysis, used the completeness-corrected values of $N_{\text {ref }}$ as above.

Table 2 shows the average and maximal values of the ratio $N_{\text {ref }} / N_{\text {at }}$ in different resolution shells. In a number of shells the maximal value exceeds the average values more than the variation of the Matthews coefficient would allow according to (6). This happens often for crystals with a high local symmetry, in particular for crystals of viruses. One reason is the presence of coordinates for only one molecule of several linked by a local symmetry, similar to the $2 \mathrm{v} 5 \mathrm{k}$ and 1 yqn cases (see \$3.1). Another reason is missed atoms in disordered parts or domains. We choose not to eliminate or correct these structures as to do so could involve multiple subjective choices.

The logarithm of the minimal ratio $N_{\text {ref }} / N_{\text {at }}$ for resolutions up to $2.5 \AA$ closely follows the line with slope equal to -3 (Fig. 2). Corresponding crystals have a $V_{\mathrm{M}}(2)$ close to $1.5 \AA^{3} \mathrm{Da}^{-1}$. For comparison, Fig. 2 also shows the straight line for crystals with $V_{\mathrm{M}}=$ $2.4 \AA^{3} \mathrm{Da}^{-1}$, as in Fig. 1.

Fig. 2 also shows that at resolutions greater than $2.5 \AA$ the logarithm of the average value $\left\langle N_{\text {ref }} / N_{\text {at }}\right\rangle$ is a quasi-linear function of the logarithm of the resolution, $\ln d_{\mathrm{PDB}}$. As expected from Fig. 1, the slope of this line differs from those of the lines corresponding to the $V_{\mathrm{M}}$ constant. This agrees with the previous demonstration by Kantardjieff \& Rupp (2003) 
Table 3

Coefficients of the linear approximations.

Each function $f(d)$ is presented as a linear function of the resolution logarithm, $f(d)=a \ln d+b$. Data sets (column 2) are defined in the text. Column 3 shows the resolution interval used to calculate the linear interpolation. Columns 6 and 8 show the root-mean-square-deviation values for the interpolation and extrapolation intervals.

\begin{tabular}{llllllll}
\hline Function $f(d)$ & $\begin{array}{l}\text { Data } \\
\text { set }\end{array}$ & $\begin{array}{l}\text { Interpolation } \\
\text { interval }\end{array}$ & $a$ & $b$ & $\begin{array}{l}\text { R.m.s.d. } \\
\text { interpolation }\end{array}$ & $\begin{array}{l}\text { Extrapolation } \\
\text { interval }\end{array}$ & $\begin{array}{l}\text { R.m.s.d. } \\
\text { extrapolation }\end{array}$ \\
\hline $\ln \left(\left\langle N_{\text {ref }} / N_{\text {at }}\right\rangle\right)$ & 2 & $0.76-2.58$ & -2.31 & 3.91 & 0.0413 & $0.76-4.42$ & 0.4503 \\
$\ln \left[\mu\left(N_{\text {ref }} / N_{\text {at }}\right)\right]$ & 2 & $0.76-2.58$ & -2.23 & 3.85 & 0.0490 & $0.76-4.42$ & 0.0884 \\
$\ln \left[\mu\left(N_{\text {ref }} / N_{\text {at }}\right)\right]$ & 2 & $0.76-2.58$ & -2.23 & 3.85 & 0.0490 & $0.76-5.06$ & 0.1031 \\
$\ln \left[\mu\left(N_{\text {ref }} / N_{\text {at }}\right)\right]$ & 2 & $0.76-4.42$ & -2.25 & 3.83 & 0.0701 & $0.76-5.06$ & 0.0732 \\
$\ln \left[\mu\left(N_{\text {ref }} / N_{\text {at }}\right)\right]$ & 2 & $0.76-4.42$ & -2.25 & 3.83 & 0.0701 & $0.76-2.58$ & 0.0580 \\
$\ln \left[\mu\left(N_{\text {ref }} / N_{\text {at }}\right)\right]$ & 3 & $0.87-3.37$ & -2.10 & 3.68 & 0.0910 & & 0.0125 \\
$\left\langle R_{\text {PDB }}\right\rangle$ & 4 & $0.87-3.86$ & 0.0874 & 0.1386 & 0.0065 & $0.76-5.06$ & 0.0249 \\
$\left\langle R_{\text {PDB }}\right\rangle$ & 4 & $0.76-5.06$ & 0.0992 & 0.1339 & 0.0102 & $0.60-10.0$ & 0.0109 \\
$\mu\left(R_{\text {PDB }}\right)$ & 4 & $0.87-3.86$ & 0.0912 & 0.1343 & 0.0098 & $0.76-5.06$ & 0.0109 \\
$\mu\left(R_{\text {PDB }}\right)$ & 4 & $0.76-5.06$ & 0.0943 & 0.1306 & 0.0107 & & 0.0088 \\
$\mu(R)$ & 1 & $0.87-3.86$ & 0.0716 & 0.1560 & 0.0076 & $0.76-5.06$ & \\
$\mu(R)$ & 1 & $0.76-5.06$ & 0.0695 & 0.1599 & 0.0085 & & \\
$\mu(R)$ & 5 & $0.87-3.86$ & 0.0804 & 0.1470 & 0.0070 & & \\
$\mu\left(R_{\text {free }}\right)$ & 5 & $0.87-3.86$ & 0.1050 & 0.1672 & 0.0069 & & \\
$\mu\left(R_{\text {free }}-R\right)$ & 5 & $0.87-2.95$ & 0.0238 & 0.0201 & 0.0022 & & \\
$\mu\left(R_{\text {PDBmin }}\right)$ & 4 & $0.60-2.95$ & 0.0163 & 0.0884 & 0.0089 & & \\
$\mu\left(R_{\text {PDBmin }}\right)$ & 4 & $2.95-6.63$ & 0.2859 & -0.2006 & 0.0118 & & \\
\hline
\end{tabular}

most frequent $N_{\text {ref }} / N_{\text {at }}$ for a given resolution, the mode $\mu\left(N_{\text {ref }} / N_{\text {at }}\right)$, are much less sensitive to outliers.

For resolution shells better than $0.8 \AA$ or worse than $4.4 \AA$ the number of available structures is low and thus the statistics are relatively poor. For other shells the distribution of $N_{\text {ref }} / N_{\text {at }}$ is essentially unimodal, with a relatively symmetric peak for the most frequent values (Fig. 3; see also the relevant Fig. 3 in Kantardjieff \& Rupp, 2003). In the resolution shells between approximately 0.9 and $2.5 \AA$ the mode $\mu\left(N_{\text {ref }} /\right.$ $N_{\text {at }}$ ) essentially coincides with $\left\langle N_{\text {ref }} / N_{\text {at }}\right\rangle$ (Fig. 2). For lower resolutions of up to $4.4 \AA\left\langle N_{\text {ref }} / N_{\text {at }}\right\rangle$ deviates from the straight line while the mode $\mu\left(N_{\text {ref }} / N_{\text {at }}\right)$ continues following it. In fact, even in the intervals with relatively poor statistics, 4.4-5.1 and 0.67-0.76 $\AA$, the most frequent values of $N_{\text {ref }} / N_{\text {at }}$ also follow this straight line (Fig. 3, Table 3).

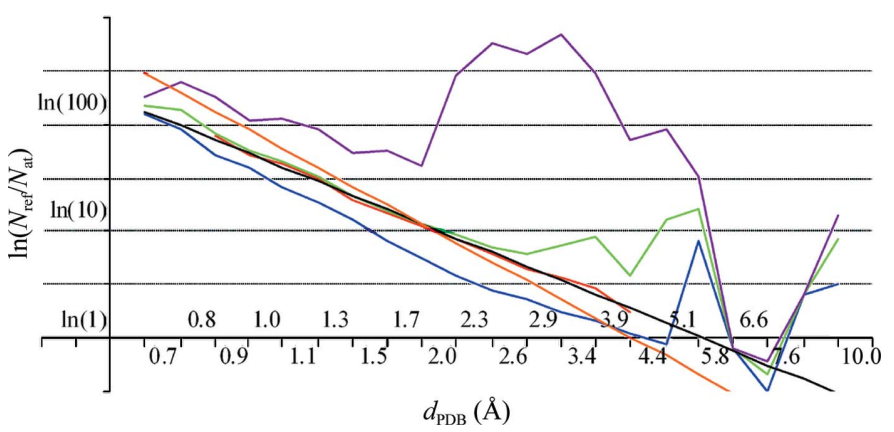

Figure 2

Logarithm $\ln \left(N_{\text {ref }} / N_{\text {at }}\right)$ as a function of resolution $d_{\mathrm{PDB}}$ on a uniform logarithmic scale. The curves show the minimal (blue), maximal (violet), average (green) and mode (red) values for the protein structures reported in the PDB (set 2). The mode line is shown as the interval in which this value was calculated. The straight line in orange is the same as in Fig. 1 showing the ratio for crystals with $V_{\mathrm{M}}=2.4 \AA^{3} \mathrm{Da}^{-1}$. The black line shows the linear interpolation to the mode.

that on average the lower the resolution of the crystals, the larger the Matthews coefficient [these authors also made a linear regression analysis for $V_{\mathrm{M}}\left(d_{\mathrm{PDB}}\right)$ using an intuitive resolution scale]. Table 3 gives the coefficients of the corresponding linear approximation performed in the interval (0.8 $\mathrm{A}, 2.6 \AA$ ) and the r.m.s.d. (root-mean-square deviation) from it. One can observe that for a few structures reported with an upper diffraction limit of between 5.8 and $7.6 \AA$ the points for their $\left\langle N_{\text {ref }} / N_{\text {at }}\right\rangle$ also fall on this line.

\subsection{Studies of the mode}

Outliers with a very large $N_{\text {ref }} / N_{\text {at }}$ may influence the $\left\langle N_{\text {ref }} /\right.$ $\left.N_{\text {at }}\right\rangle$ values. For example, $\left\langle N_{\text {ref }} / N_{\text {at }}\right\rangle$ significantly fluctuates at low resolution (see discussion above). At the same time, the other characteristics of a distribution such as the values of the
The corresponding linear interpolation (Table 3 ) allows the 'most typical $N_{\text {ref }} / N_{\text {at }}$ value at a given resolution' to be estimated analytically as

$$
\mu_{\text {prot }}\left(N_{\text {ref }} / N_{\text {at }}\right) \simeq 45.1 d_{\mathrm{PDB}}^{-2.25}
$$

Table 2 shows interpolated and extrapolated values together with experimentally obtained values.

For crystals of nucleic acids without proteins the behaviour is quite similar (details not shown) even though the statistics are much poorer owing to the small sample size (set 3; Table $1)$. The linear approximation of the mode $\mu_{\text {nucl }}\left(N_{\text {ref }} / N_{\text {at }}\right)$,

$$
\mu_{\text {nucl }}\left(N_{\text {ref }} / N_{\text {at }}\right) \simeq 39.6 d_{\mathrm{PDB}}^{-2.10}
$$

differs only slightly from that obtained for proteins (Table 3 ).

\subsection{Possible applications}

This simple behaviour of typical $N_{\text {ref }} / N_{\text {at }}$ values over a wide resolution range may be helpful for existing tools, for example Matthews Probability Calculator (Kantardjieff \& Rupp, 2003) or phenix.xtriage (Zwart et al., 2005), especially at extreme resolutions. Combining (6) and (7) gives a simple analytic estimation

$$
V_{\mathrm{M}}=\frac{1}{\eta} 45.1 d_{P D B}^{0.75} \simeq 1.67 d_{\mathrm{PDB}}^{0.75} .
$$

Inverting (9), one can estimate the limit

$$
d_{\mathrm{PDB}} \simeq\left(0.60 V_{\mathrm{M}}\right)^{1.33} \simeq 0.506 V_{\mathrm{M}}^{1.33}
$$

to which a crystal with a given $V_{\mathrm{M}}$ is expected to diffract. This information could be taken into account when considering how much effort should be applied to obtaining improved diffraction data from a given crystal form of a specific protein. Obviously, (10) only provides a typical limit, while better results may be obtained for a particular crystal. As an 
example, human aldose reductase crystals have a $V_{\mathrm{M}}$ of $2.10 \AA^{3} \mathrm{Da}^{-1}$, giving an estimated $d_{\mathrm{PDB}}$ of $\sim 1.35 \AA$. This confirms that the value of $1.7 \AA$ initially reported at a home source (Lamour et al., 1999) was below what might be obtained. At the same time, (10) does not predict that some aldose reductase crystals can diffract to $0.66 \AA$ resolution (Howard et al., 2004). Nevertheless, the possibility of similarly high-resolution data can be predicted for other crystals. An example is the polypeptide YGG crystal (Pichon-Pesme et al., $2000 ; V_{\mathrm{M}}=1.12 \AA^{3} \mathrm{Da}^{-1}$ ) for which (10) gives $d_{\mathrm{PDB}} \simeq 0.60 \AA$. Indeed, for this crystal the $50 \%$ completeness data set was measured at $0.59 \AA$ resolution (the highest resolution reflection measured was at $0.44 \AA$ resolution).

The predictability of the typical $N_{\text {ref }} / N_{\text {at }}$ values suggests the definition of the maximal number of parameters per atom that are 'usual at a given resolution', avoiding overparametrization (Table 2). In other words, this defines the number of atomic parameters that can typically be used at a given resolution. While for a particular model the number $N_{\text {ref }} / N_{\text {at }}$ can be calculated precisely at any given resolution, knowledge of

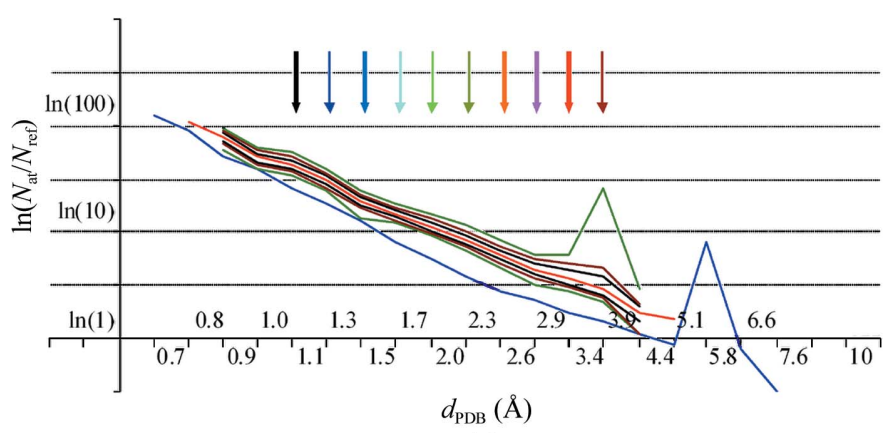

(a)

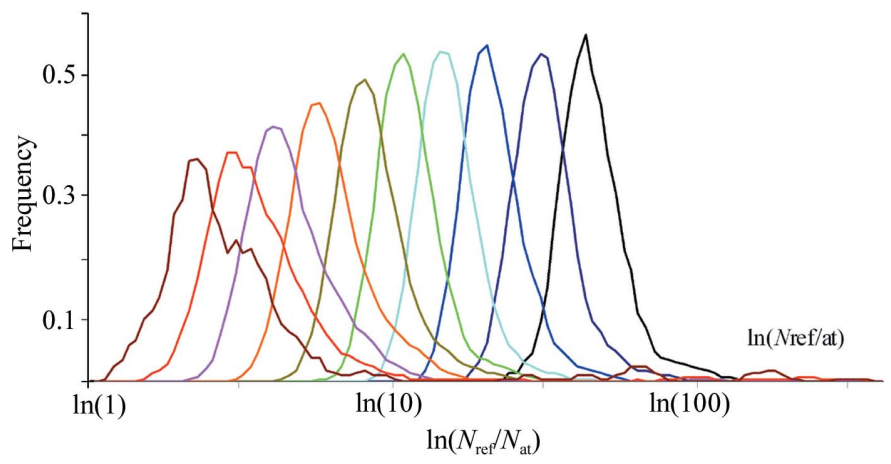

(b)

\section{Figure 3}

(a) The mode $\mu\left(N_{\text {ref }} / N_{\text {at }}\right)$ as a function of the resolution $d_{\mathrm{PDB}}$ on a uniform logarithmic scale. The thick red curve shows the mode values as a function of resolution on a uniform logarithmic scale for the protein structures reported in the PDB (set 2). The thin lines show, as corridors, the distribution of the models around the mode. Each corridor contains $40 \%$ (black), $60 \%$ (brown) and $80 \%$ (dark green), respectively, of the structures in the corresponding resolution shell, half above and half below the mode. The corridors are shown at a resolution interval with a high enough number of models to calculate these values; the mode was formally calculated and is also shown for one higher resolution interval and one lower resolution interval even when the statistics there were poor. The blue line shows the minimal values for comparison (Table 1). Coloured arrows correspond to the distributions shown in $(b)$. (b) Distribution of $N_{\text {ref }} / N_{\text {at }}$ for several selected resolutions as indicated by coloured arrows in $(a)$. typical values is crucial for software and methods developers, allowing them to automate model-refinement protocols. In particular, the ratios of 4 and 10 at resolutions of approximately 3 and $2 \AA$, respectively, give the minimal theoretical limits at which individual isotropic or anisotropic displacement parameters can be used (with four or ten parameters per atom, respectively). Obviously, in these cases the ratio $N_{\text {ref }} / N_{\text {at }} \simeq 1$ and therefore in practice higher resolution limits are recommended even when various restraints are introduced. The possibility of unrestrained refinement is not surprising at $1 \AA$ or higher, where there are four reflections per parameter even for an anisotropic model. A very high ratio of above 80 at resolutions better than $0.8 \AA$ leads one to believe that the diffraction data will contain a lot of additional information (as confirmed by residual maps) and that a more detailed model is required. At the low-resolution end, the typical ratio prescribes the size of rigid groups that can realistically be introduced.

\section{4. $R$ factors on a logarithmic scale}

\subsection{PDB-reported $\boldsymbol{R}$ factors}

While $N_{\text {ref }} / N_{\text {at }}$ characterizes the amount of 'diffraction information' at a given resolution and defines the type of model, the crystallographic $R$ factor is a conventional measure

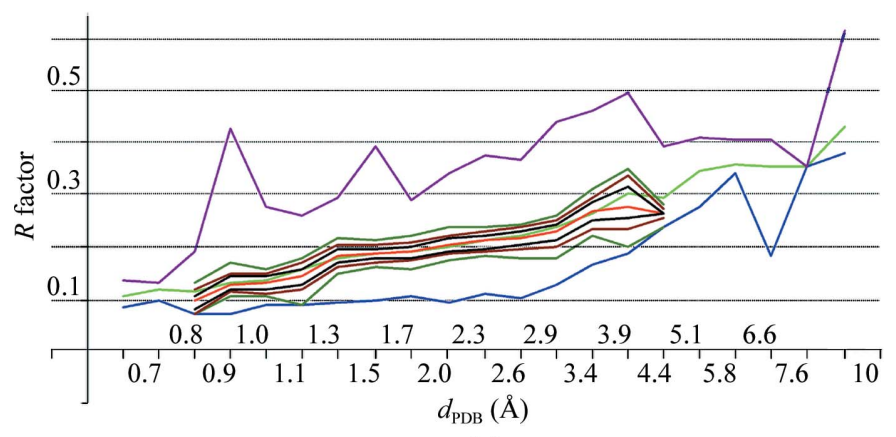

(a)

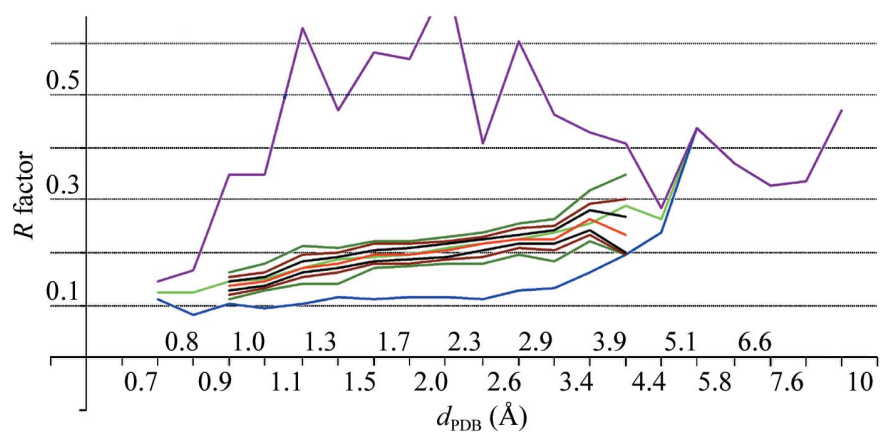

(b)

Figure 4

$R$ factors as a function of resolution $d_{\mathrm{PDB}}$ on a logarithmic scale. The curves show the minimal (blue), average (green), maximal (violet) and mode (red) values; the mode is calculated in the intervals containing a high enough number of models. The thin lines show the corridors around the mode. Each corridor contains $40 \%$ (black), $60 \%$ (brown) and $80 \%$ (dark green) of the structures, respectively, in the corresponding resolution shell, half above and half below the mode. (a) $R$ factors reported in the PDB; set 4 of models. (b) $R$ factors recalculated with phenix. model_vs_data; set 5 of models. 
Table 4

Statistical information for the $R$ factors in resolution shells chosen uniformly on the logarithmic scale.

Columns 2, 3 and 4 give the PDB codes for the models with the minimal $R$-factor values reported in the PDB headers and recalculated by phenix.model_vs_data $(\mathrm{mvd})$. Linear interpolations are given for the mode of corresponding values calculated for sets 4 (column 5) and set 5 (columns 6-8).

\begin{tabular}{|c|c|c|c|c|c|c|c|}
\hline \multirow[b]{2}{*}{$\begin{array}{l}\text { Resolution } \\
\text { shell }(\AA)\end{array}$} & \multicolumn{3}{|c|}{ PDB code } & \multicolumn{4}{|c|}{ Linear interpolation } \\
\hline & $\begin{array}{l}\text { Min. } R \\
\text { (PDB) }\end{array}$ & $\begin{array}{l}\text { Min. } R \\
(\mathrm{mvd}, \text { set } 4)\end{array}$ & $\begin{array}{l}\text { Min. } R \\
(\mathrm{mvd}, \text { set 5) }\end{array}$ & $\mu\left(R_{\mathrm{PDB}}\right)$ & $\mu(R)$ & $\mu\left(R_{\text {free }}\right)$ & $\mu\left(R_{\text {free }}-R\right)$ \\
\hline$<0.67$ & $2 \mathrm{vb} 1$ & $2 \mathrm{vb} 1$ & - & 0.0911 & 0.1090 & 0.1175 & 0.0089 \\
\hline $0.67-0.76$ & $1 \mathrm{yk} 4$ & $1 \mathrm{r} 6 \mathrm{j}$ & $2 \mathrm{pve}$ & 0.1035 & 0.1199 & 0.1317 & 0.0121 \\
\hline $0.76-0.87$ & $2 \mathrm{ol} 9$ & $2 \mathrm{~h} 5 \mathrm{c}$ & $2 \mathrm{~h} 5 \mathrm{c}$ & 0.1158 & 0.1307 & 0.1459 & 0.0153 \\
\hline $0.87-1.00$ & $1 \mathrm{ob} 7$ & $1 \mathrm{rb} 9$ & $1 \mathrm{ixb}$ & 0.1281 & 0.1416 & 0.1601 & 0.0185 \\
\hline $1.00-1.14$ & 1iro & 1iro & $1 \mathrm{z} 3 \mathrm{n}$ & 0.1405 & 0.1525 & 0.1742 & 0.0217 \\
\hline $1.14-1.31$ & $2 \mathrm{v} 91$ & $1 \mathrm{n} 0 \mathrm{q}$ & $2 \mathrm{v} 91$ & 0.1528 & 0.1634 & 0.1884 & 0.0250 \\
\hline $1.31-1.50$ & $1 \mathrm{hbz}$ & $2 \mathrm{plz}$ & $1 \mathrm{hbz}$ & 0.1651 & 0.1743 & 0.2026 & 0.0282 \\
\hline $1.50-1.72$ & $2 \mathrm{ah} 2$ & 6rxn & $2 \mathrm{pfg}$ & 0.1775 & 0.1851 & 0.2168 & 0.0314 \\
\hline $1.72-1.97$ & $1 \mathrm{amk}$ & 2dya & 2dya & 0.1898 & 0.1960 & 0.2310 & 0.0346 \\
\hline $1.97-2.25$ & 2oh5 & $2 \mathrm{oh} 5$ & $2 \mathrm{oh} 5$ & 0.2021 & 0.2069 & 0.2452 & 0.0378 \\
\hline $2.25-2.58$ & 2oh7 & 1uvw & 1uvw & 0.2145 & 0.2178 & 0.2594 & 0.0410 \\
\hline $2.58-2.95$ & 5 bna & 1tre & $1 \mathrm{f} 4 \mathrm{~h}$ & 0.2268 & 0.2286 & 0.2736 & 0.0443 \\
\hline $2.95-3.37$ & $1 \mathrm{bgj}$ & 1sv2 & $1 \mathrm{ydz}$ & 0.2391 & 0.2395 & 0.2878 & 0.0475 \\
\hline $3.37-3.86$ & $2 \mathrm{~d} 3 \mathrm{~b}$ & $1 \mathrm{gn} 3$ & $2 q 3 n$ & 0.2515 & 0.2504 & 0.3020 & 0.0507 \\
\hline $3.86-4.42$ & 1aos & 1 veq & 1 veq & 0.2638 & 0.2613 & 0.3162 & 0.0539 \\
\hline $4.42-5.06$ & $2 \mathrm{rkj}$ & $1 \mathrm{pgf}$ & 2rkj & 0.2761 & 0.2721 & 0.3304 & 0.0571 \\
\hline $5.06-5.80$ & $3 b 5 w$ & $2 \mathrm{~b} 66$ & $3 b 5 x$ & 0.2885 & 0.2830 & 0.3445 & 0.0603 \\
\hline $5.80-6.63$ & $2 b 9 n$ & $2 b 9 n$ & $3 e 3 j$ & 0.3008 & 0.2939 & 0.3587 & 0.0635 \\
\hline $6.63-7.59$ & $3 c 4 y$ & $3 c 4 y$ & $1 \mathrm{yv} 0$ & 0.3131 & 0.3048 & 0.3729 & 0.0668 \\
\hline $7.59-8.69$ & $2 \mathrm{dh} 1$ & $2 \mathrm{dh} 1$ & $2 \mathrm{dh} 1$ & 0.3255 & 0.3157 & 0.3871 & 0.0700 \\
\hline $8.69-9.95$ & $1 \mathrm{vcr}$ & $1 \mathrm{zbb}$ & $1 \mathrm{vcr}$ & 0.3378 & 0.3265 & 0.4013 & 0.0732 \\
\hline
\end{tabular}

reported $R_{\mathrm{PDB}}$ represented values other than the conventional $R$ factor (for all these entries the value was below 0.06$)$. For other entries, excluding a nonmacromolecular model of actinomycin (PDB code 1a7y; Schäfer et al., 1998; $\left.R_{\mathrm{PDB}}=0.058\right)$, the reported value $R_{\mathrm{PDB}}$ varied between 0.072 and 0.615 . Exluding actinomycin, we arrived at a total of 30546 models (set 4; Table 1).

The same resolution intervals with an equal length on the logarithmic scale were used as defined in $\$ 2$. Resolution shells at very high and low resolutions had poor statistics. In each of the other resolution shells the distribution of $R$ factors was unimodal, with a clear value for the mode $\mu\left(R_{\mathrm{PDB}}\right)$. In all shells up to the resolution shell 3.0-3.5 $\AA$ the peaks were more or less symmetric and quite narrow. The intervals $\left[\mu\left(R_{\mathrm{PDB}}\right)-\delta\right.$, $\left.\mu\left(R_{\mathrm{PDB}}\right)+\delta\right]$ contained nearly 40,60 or $80 \%$ of the structures reported at this resolution $d_{\mathrm{PDB}}$ when $\delta=0.01,0.02$ or 0.03 , respectively (Fig. $4 a$ ). Where calculated, $\mu\left(R_{\mathrm{PDB}}\right)$ is close to the average value $\left\langle R_{\mathrm{PDB}}\right\rangle$.

It is has previously been observed that $\left\langle R_{\mathrm{PDB}}\right\rangle$ increases with resolution and that this growth is nonlinear on a uniform scale in angstroms (see, for example, Read \& Kleywegt, 2009; Joosten et al., 2009). However, it is practically linear up to $3.5 \AA$ when the resolution is expressed on the logarithmic scale, as is $\mu\left(R_{\mathrm{PDB}}\right)$ (Fig. 5). Table 3 gives the coefficients of the corresponding linear interpolations (Table 4). The r.m.s.d. of the interpolation

$$
\mu\left(R_{\mathrm{PDB}}\right) \simeq 0.091 \ln d_{\mathrm{PDB}}+0.134
$$

Figure 5

Linear approximation to the $R$ factors. The red and blue curves show the mode and minimal values for the $R$ values extracted from the PDB headers. The curves in magenta and in green show the mode value for the $R_{\text {free }}$ factor and for the difference factor $\Delta R=R_{\text {free }}-R$ recalculated for set 5 of models. The straight lines in brown, black, violet and dark green illustrate the corresponding linear approximations (Table 3). The line in light blue shows the mode for the $R$ factor recalculated for the largest possible set of models (set 4). The curves are shown for resolution shells containing a high enough number of models to calculate the values.

of the diffraction quality of these models, although it is not fully reliable as indicated in a series of papers starting with Brändén \& Jones (1990). There are anecdotal 'rules of thumb' for acceptable values. We searched for a simple dependence of $R$ factors on the resolution, substituting the usual uniform resolution scale by a uniform logarithmic scale.

For our analysis we took the same full set of 31662 models (set 1) as above. We excluded 1088 entries with an incorrectly reported value of the $R$ factor $\left(R_{\mathrm{PDB}}\right)$. We also removed 15 structures with $R_{\mathrm{PDB}}>17.0$ (probably reported as a percentage and not as a fraction) and 11 models for which the in the interval $(0.87,3.86)$ does not change on including $\mu\left(R_{\mathrm{PDB}}\right)$ values for lower and higher resolution intervals with poorer statistics.

Interestingly, the minimal values $R_{\mathrm{PDBmin}}$ are practically constant at around 0.10 in all resolution shells up to $2.6 \AA$ (Fig. 4a). In other words, at all these resolutions it is possible to obtain a conventional atomic model reproducing the experimental diffraction data with a similar and sufficiently small relative error ( $R$ factor). The approach of $\mu\left(R_{\mathrm{PDB}}\right)$ and $\left\langle R_{\mathrm{PDB}}\right\rangle$ to 0.10 at near-atomic resolutions of $\sim 1 \AA$ and the statistically significant number of reported models means that here most of the models achieve this high quality. The increase in $\mu\left(R_{\mathrm{PDB}}\right)$ with resolution from 1 to $3 \AA$ indicates that while it is still possible to obtain a high-quality model, this requires more and more high-quality data, particular effort and luck. Below $2.6 \AA$ resolution $R_{\text {PDBmin }}$ starts to grow sharply. At a similar resolution, the minimal Matthews coefficient of known macromolecular crystals also starts growing as indicated by changing the slope of the curve $\min \ln \left(N_{\text {ref }} / N_{\text {at }}\right)$ (Fig. 2). 
In $\$ 5$ we speculate about the possible meaning of the intersection of the straight lines for $\left\langle R_{\mathrm{PDB}}\right\rangle$ and $\mu\left(R_{\mathrm{PDB}}\right)$ with the curve for $R_{\text {PDBmin }}$ at resolutions of $\sim 0.7-0.8 \AA$ and $\sim 6 \AA$.

\subsection{Recalculated $R$ factors}

In order to remove errors and inconsistencies in $R_{\mathrm{PDB}}$ other than those indicated above in $\$ 4.1$, we recalculated the $R$-factor value for all 32662 structures using the phenix. model_vs_data tool of PHENIX. Extremely high or unreasonably low values of the calculated $R$ factor indicated some inconsistency between the reported models or data. In spite of these obvious outliers, the general behaviour of the $R$ factor was similar to that for $R_{\mathrm{PDB}}$ [details not shown; see Fig. 5 for the mode $\mu(R)$ values]. For some models the obtained $R$ values were slightly higher than $R_{\mathrm{PDB}}$, while for others they were lower. The details of this comparison will be reported elsewhere. In general, the average difference is within reasonable limits. It is slightly positive at higher resolutions $\left(d_{\mathrm{PDB}}<1.2 \AA\right)$, where for a number of models it was impossible to reproduce accurately the authors' calculations.

We chose not to remove outliers using $\sigma$ or outlier cutoff levels, the choice of which is subjective. Instead, we repeated the calculations with a subset containing the entries for which the test data sets were available and the $R_{\text {free }}$ value could be calculated (set 5; 22504 models). Here, all models had $0.082 \leq$ $R \leq 0.626$, with a single exception $(R=0.715)$; thus, outliers did not strongly influence the average and especially the mode values (Fig. 4b).

Qualitatively, the behaviour of the $R$ factor for both sets of models (sets 4 and 5) is similar to that of $R_{\mathrm{PDB}}$. For the recalculated $R$ factors, which are unbiased by the diversity of protocols and software, the mode $\mu(R)$ is a quasi-linear function of $\ln d_{\mathrm{PDB}}$ in the whole resolution range in which it was calculated (up to $4.4 \AA$ ). For the reasons mentioned above this line has a slope that is slightly lower (Table 3 ) than that for $\mu\left(R_{\mathrm{PDB}}\right)$.

\section{3. $R_{\text {free }}$ and difference $R_{\text {free }}-R$}

In general, the $R_{\text {free }}$ calculated for set 5 of the PDB entries behaved similarly to $R$. On the logarithmic scale $\left\langle R_{\text {free }}\right\rangle$ is quasi-linear up to a resolution of $4 \AA$. The same was observed for $\mu\left(R_{\text {free }}\right)$ in all intervals in which it was possible to calculate it (Fig. 5). Table 3 gives the coefficients of the corresponding linear approximation (Table 4).

The difference $\Delta R=R_{\text {free }}-R$, which is useful for model validation, is on average positive as expected (Brünger, 1992). All resolution shells contained obvious outliers with $\Delta R$ close to 0 or even negative. The mode values $\mu(\Delta R)$ are independent of these outliers and therefore we did not exclude them by subjective cutoffs. These characteristics are practically linear at resolutions higher than $3 \AA$ (Fig. 5). This makes it possible to suggest a simple formula for the $\Delta R$ typical at a given resolution $d_{\mathrm{PDB}}$ (Table 3 ),

$$
\mu(\Delta R) \simeq 0.024 \ln d_{\mathrm{PDB}}+0.020
$$

At resolutions below $3 \AA$ the difference $\mu(\Delta R)$ is lower than that predicted by (12). On one hand, there is no proof that (12) should be applicable at all resolutions. On the other, there are a number of hypothetical reasons that could decrease the reliability of $R_{\text {free }}$ statistics for low resolutions. For example, a smaller number of reflections may make test sets and corresponding statistics poorer, reflections from the test sets may be indirectly related to those from the work sets for structures with local symmetries (Fabiola et al., 2006; as discussed in $\$ 3.2$, such structures are more frequent at lower resolutions) etc.

\section{Discussion}

A nonlinear rescaling of a function or its argument(s) modifies the shape of its plot and a judicious choice of scale may help to clarify the dependence. Obviously, the simplest dependence is a linear dependence, which can even be identified visually. In crystallography, many characteristics are functions of resolution. The resolution scale is usually linear, quadratic or cubic, either in direct or in reciprocal space, or chosen in some other intuitive way. The logarithmic scale we have described naturally increases the number of reflections by a given factor from one resolution limit to another when the limits are chosen uniformly. In our study we have analyzed several crystallographic characteristics as a function of the resolution $d_{\mathrm{PDB}}$ at which structures have been reported. In contrast to traditional studies of the mean values of functions, we analyzed their modes $\mu$ (most frequent values), which are less sensitive to outliers, although in many cases the conclusions are also applicable to the mean values.

The ratio $N_{\text {ref }} / N_{\text {at }}$ of the number of independent reflections to the number of independent macromolecular non-H atoms in the unit cell is an important characteristic of structural projects. It is an appropriate candidate for study using a logarithmic scale because of the cubic dependence of $N_{\text {ref }} / N_{\text {at }}$ on $d_{\mathrm{PDB}}$ for crystals with the same Matthews coefficient. A derived dependence of $\mu\left(N_{\text {ref }} / N_{\text {at }}\right)$ on $d_{\mathrm{PDB}}$ with a power close to -2.2 was easily observed when using the logarithmic scale and is difficult to deduce otherwise. This dependence can be used to help define the upper limits on the parameterization of macromolecule models possible at a given resolution. It may also be used to help to predict the number of molecules in the unit cell or to estimate the expected diffraction limit of a crystal.

Using a logarithmic scale to study $R$ factors is less intuitive. However, in contrast to previous studies using traditional scales, here quasi-linear behaviour was observed for the mode of $R$ factors both reported in the PDB and recalculated from the models and data. Similarly, the mode for $R_{\text {free }}$ and the difference between $R$ factors are linear at resolutions better than $3 \AA$. Corresponding linear approximations can be used to help to guide refinement and validation of atomic models.

Interestingly, the two points of the intersection of the straight line for $\mu(R)$ with the curve for $R_{\min }$ have common features. They both mark limits where correcting terms to the structure factors of a conventional independent-atoms model $\left(F_{\text {IAM }}\right)$, 


$$
F_{\text {model }}=F_{\text {IAM }}+F_{\text {IAS }}+F_{\text {bulk-solvent }} \text {, }
$$

become crucial: a bulk-solvent contribution $F_{\text {bulk-solvent }}$ (see, for example, Jiang \& Brünger, 1994) below the low-resolution limit of $\sim 6 \AA$ and density-deformation structure factors $F_{\text {IAS }}$ (for example, using interatomic scatterers; Afonine et al., 2004) at ultrahigh resolution, i.e. higher than approximately $0.7 \AA$ A. Efficient bulk-solvent (Afonine et al., 2005) and IAS corrections (Afonine et al., 2007) are available in PHENIX. We conclude that these resolution extremes mark points at which features of the electron density are not well modelled by single isotropic or anisotropic scatterers centred on the atomic positions.

We postulate that other crystallographic phenomena can be uncovered using a uniform logarithmic scale. For example, the peak distribution in the averaged and individual $|E(d)|$ profiles (Morris \& Bricogne, 2003; Morris et al., 2004) is more or less uniform when using a logarithmic scale. However, at present we cannot determine whether this is purely coincidental or the result of some underlying physical meaning.

PDA would like to thank NIH/NIGMS for generous support of the PHENIX project (1P01 GM063210). This work was supported in part by the US Department of Energy under contract No. DE-AC02-05CH11231.

\section{References}

Adams, P. D., Grosse-Kunstleve, R. W., Hung, L.-W., Ioerger, T. R., McCoy, A. J., Moriarty, N. W., Read, R. J., Sacchettini, J. C., Sauter, N. K. \& Terwilliger, T. C. (2002). Acta Cryst. D58, 1948-1954.

Afonine, P. V., Grosse-Kunstleve, R. W. \& Adams, P. D. (2005). Acta Cryst. D61, 850-855.

Afonine, P. V., Grosse-Kunstleve, R. W., Adams, P. D., Lunin, V. Y. \& Urzhumtsev, A. (2007). Acta Cryst. D63, 1194-1197.

Afonine, P. V., Lunin, V. Y., Muzet, N. \& Urzhumtsev, A. (2004). Acta Cryst. D60, 260-274.

Arai, S., Chatake, T., Suzuki, N., Mizuno, H. \& Niimura, N. (2004). Acta Cryst. D60, 1032-1039.
Bernstein, F. C., Koetzle, T. F., Williams, G. J., Meyer, E. F. Jr, Brice, M. D., Rodgers, J. R., Kennard, O., Shimanouchi, T. \& Tasumi, M. (1977). J. Mol. Biol. 112, 535-542.

Berman, H. M., Westbrook, J., Feng, Z., Gilliland, G., Bhat, T. N., Weissig, H., Shindyalov, I. N. \& Bourne, P. E. (2000). Nucleic Acids Res. 28, 235-242.

Brändén, C.-I. \& Jones, T. A. (1990). Nature (London), 343, 687-689.

Brünger, A. T. (1992). Nature (London), 355, 472-475.

Brünger, A. T. (1997). Methods Enzymol. 276, 366-396.

Cruickshank, D. W. J. (1996). Proceedings of the CCP4 Study Weekend. Refinement of Macromolecular Structures, edited by E. Dodson, M. Moore, A. Ralph \& S. Bailey, pp 11-22. Warrington: Darebury Laboratory.

Fabiola, F., Korostelev, A. \& Chapman, M. S. (2006). Acta Cryst. D62, 227-238.

Howard, E. I., Sanishvili, R., Cachau, R., Mitschler, A., Chevrier, B., Barth, P., Lamour, V., Van Zandt, M., Sibley, E., Bon, C., Moras, D., Schneider, T. R., Joachimiak, A. \& Podjarny, A. (2004). Proteins, 55, 792-804.

Jiang, J.-S. \& Brünger, A. T. (1994). J. Mol. Biol. 243, 100-115.

Joosten, R. P. et al. (2009). J. Appl. Cryst. 42, 376-384.

Kantardjieff, K. A. \& Rupp, B. (2003). Protein Sci. 12, $1865-$ 1871.

Lamour, V., Barth, P., Rogniaux, H., Poterszman, A., Howard, E., Mitschler, A., Van Dorsselaer, A., Podjarny, A. \& Moras, D. (1999). Acta Cryst. D55, 721-723.

Luzzati, V. (1952). Acta Cryst. 5, 802-810.

Matthews, B. W. (1968). J. Mol. Biol. 33, 491-497.

Morris, R. J., Blanc, E. \& Bricogne, G. (2004). Acta Cryst. D60, 227-240.

Morris, R. J. \& Bricogne, G. (2003). Acta Cryst. D59, 615-617.

Pichon-Pesme, V., Lachekar, H., Souhassou, M. \& Lecomte, C. (2000). Acta Cryst. B56, 728-737.

Read, R. J. \& Kleywegt, G. J. (2009). Acta Cryst. D65, 140-147.

Schäfer, M., Sheldrick, G. M., Bahner, I. \& Lackner, H. (1998). Angew. Chem. 37, 2381-2384.

Tickle, I. J., Laskowski, R. A. \& Moss, D. S. (1998). Acta Cryst. D54, 547-557.

Tickle, I. J., Laskowski, R. A. \& Moss, D. S. (2000). Acta Cryst. D56, 442-450.

Urzhumtseva, L., Afonine, P. V., Adams, P. D. \& Urzhumtsev, A. (2009). Acta Cryst. D65, 297-300.

Wilson, A. J. C. (1949). Acta Cryst. 2, 318-321.

Zwart, P. H., Grosse-Kunstleve, R. W. \& Adams, P. D. (2005). CCP4 Newsl. 43, contribution 7 . 